\section{ORIGINAL RESEARCH}

B. Bender

C. Mänz

A. Korn

T. Nägele

U. Klose

\title{
Optimized 3D Magnetization-Prepared Rapid Acquisition of Gradient Echo: Identification of Thalamus Substructures at 3T
}

\begin{abstract}
BACKGROUND AND PURPOSE: Because the substructures of the thalamus are not visible on standard T1- and T2-weighted MR images, planning of deep brain stimulation implantation relies on stereotactic atlas coordinates. The goal of the present work was to test whether an optimized 3D MPRAGE protocol can depict thalamus substructures.
\end{abstract}

\begin{abstract}
MATERIALS AND METHODS: After optimization of the TI to maximize contrast between gray matter and white matter, 6 healthy subjects were scanned at 3T with the optimized 3D MPRAGE. The results were compared with stereotactic atlases, and 2 expert readers trained in thalamic anatomy identified the 4 large thalamic nuclei groups.
\end{abstract}

RESULTS: There was a high agreement between the different atlases and the resulting MR images. The 4 large thalamic nuclei groups (anterior, lateral, medial, posterior) could be detected reliably. The inter-reader consistency on the size and location was $75 \%-92 \%$.

CoNCLUSIONS: The optimized 3D MPRAGE protocol improves contrast in the thalamus, and the 4 large thalamic nuclei groups can be identified with high inter-reader agreement.

\begin{abstract}
ABBREVIATIONS: $\mathrm{AC}=$ anterior commissure; $\mathrm{ACPC}=$ anterior/posterior commissure; $\mathrm{CM}$ centre médian nucleus; $L D=$ lateral dorsal nucleus; $M D=$ mediodorsal nucleus; MPRAGE magnetization-prepared rapid acquisition of gradient echo; PC = posterior commissure
\end{abstract}

$T$ here has been increasing interest in deep brain stimulation in the treatment of several neurologic diseases since the first positive experiences in humans in the early 1980 s. $^{1}$ Typical neurologic diseases treated with deep brain stimulation are Parkinson disease, dystonia, and essential tremor. ${ }^{1}$ Typical targets in the basal ganglia are the subthalamic nucleus, the internal globus pallidus, and the thalamic nuclei such as the nucleus ventralis intermedius. ${ }^{2-4}$ More recently, the initial results of larger patient groups with epilepsy treated with deep brain stimulation in the anterior thalamus complex have been published. ${ }^{5}$ More substructures of the thalamus, such as the $\mathrm{CM}$ or the parafasicular nucleus complex, have also been reported to be possible targets for deep brain stimulation in a variety of diseases. ${ }^{6-8}$ Placement of electrodes is usually planned indirectly, with coordinates taken from stereotactic atlases of the thalamus, because the substructures of the thalamus are not visible on routine MR imaging, ${ }^{9}$ and is corrected by intraoperative electrophysiologic mapping. All histologic atlases face known problems: Sections in different orientations

Received March 2, 2011; accepted after revision April 20.

From the MR Research Group, Department of Diagnostic and Interventional Neuroradiology, University Hospital Tübingen, Tübingen, Germany.

Benjamin Bender: Planning of study, literature research, MR imaging examination, identification of thalamic substructures, statistical analysis, evaluation of results (clinical and methodologic aspects), writing of paper, approval of final version. Constantin Mänz: MR imaging examination, identification of thalamic substructures, evaluation of results (clinical aspects), approval of final version. Andreas Korn: Literature research, evaluation of results (clinical aspects), approval of final version. Thomas Nägele: Planning of study, evaluation of results (clinical aspects), approval of final version. Uwe Klose: Planning of study, statistical analysis, evaluation of results (methodologic aspects), writing of paper, approval of final version.

Please address correspondence to Benjamin Bender, MD, Department of Diagnostic and Interventional Neuroradiology, Hoppe-Seyler-Str 3, 72076 Tübingen, Germany; e-mail: benjamin.bender@med.uni-tuebingen.de

http://dx.doi.org/10.3174/ajnr.A2705 are from different subjects and the distance between adjacent sections is relatively large and may vary. To improve targeting with coordinates from classic histologic atlases, a mean 3D atlas of the human thalamus has been published recently. ${ }^{10}$

Many authors have already addressed the problem of individualized depiction of thalamic substructures. Deoni et $\mathrm{al}^{11}$ were able to show that many substructures of the thalamus can be identified with a measurement at $1.5 \mathrm{~T}$, if measurement times of several hours are used. For measurements with a shorter time, a segmentation algorithm is able to identify important substructures. Still, this segmentation algorithm of Deoni et al requires seed points taken from anatomic atlases, which face the known problems. Just recently, Lemaire et $\mathrm{al}^{12}$ reported that high-resolution T1-weighted measurements can be used to create images of the thalamus, which were very comparable to myelin-stained histologic sections. Such a contrast can easily be used to identify many well-known structures in individual thalami; however, the measurement time for the protocol used was during 14 hours. Kanowski et $\mathrm{al}^{13}$ showed that the CM is already identifiable in a reasonable measurement time of 13-26 minutes with proton-attenuation weighted images at $3 \mathrm{~T}$. The goal of the present work was to develop an MR imaging protocol at 3T that depicts the major substructures of the thalamus within a reasonable measurement time. Because T1-weighted images at high field strengths show a contrast comparable with myelin stains, ${ }^{12}$ a $3 \mathrm{D}$ MPRAGE protocol was optimized.

\section{Materials and Methods}

After approval by the local ethics committee, 6 healthy subjects ( 2 men, 4 women; 21-34 years of age; mean, 26.5 years) who gave written informed consent were included in this study. All subjects were measured with standard single-slab 3D MPRAGE sequences on a 3T scan- 


\begin{tabular}{|c|c|c|c|c|c|c|c|c|c|c|}
\hline \multicolumn{11}{|c|}{ Summary of the volume size of the thalamus and substructures of the thalamus in all subjects ${ }^{\mathrm{a}}$} \\
\hline \multirow{3}{*}{$\begin{array}{l}\text { Subject No., } \\
\text { Age (yr), } \\
\text { Sex }\end{array}$} & \multicolumn{10}{|c|}{ Size $\left(\mathrm{mm}^{2}\right)$} \\
\hline & \multicolumn{2}{|c|}{ Anterior } & \multicolumn{2}{|c|}{ Medial } & \multicolumn{2}{|c|}{ Lateral } & \multicolumn{2}{|c|}{ Posterior } & \multicolumn{2}{|c|}{ Thalamus } \\
\hline & B.B. & C.M. & B.B. & C.M. & B.B. & C.M. & B.B. & C.M. & B.B. & C.M. \\
\hline 1) $27, \mathrm{~F}$ & 218 & 203 & 1608 & 1639 & 2193 & 2152 & 2273 & 2261 & 6292 & 6255 \\
\hline 2) $26, \mathrm{M}$ & 227 & 205 & 1562 & 1476 & 2123 & 2134 & 2277 & 2291 & 6189 & 6106 \\
\hline 3) $21, \mathrm{~F}$ & 221 & 202 & 1567 & 1520 & 1969 & 1970 & 2186 & 2187 & 5943 & 5879 \\
\hline 4) $23, \mathrm{~F}$ & 200 & 186 & 1211 & 1184 & 1699 & 1740 & 1811 & 1934 & 4921 & 5044 \\
\hline 5) $34, \mathrm{~F}$ & 293 & 286 & 1555 & 1499 & 2216 & 2287 & 2008 & 2099 & 6072 & 6171 \\
\hline 6) $28, \mathrm{M}$ & 197 & 209 & 1328 & 1380 & 1728 & 1757 & 2057 & 2174 & 5310 & 5520 \\
\hline Mean \pm Std & $226 \pm 35$ & $215 \pm 32$ & $1471 \pm 162$ & $1450 \pm 141$ & $1988 \pm 230$ & $2007 \pm 204$ & $2102 \pm 180$ & $2158 \pm 118$ & $5788 \pm 548$ & $5829 \pm 426$ \\
\hline Atlas & \multicolumn{2}{|c|}{249} & \multicolumn{2}{|c|}{1380} & \multicolumn{2}{|c|}{1727} & \multicolumn{2}{|c|}{1813} & \multicolumn{2}{|c|}{5169} \\
\hline
\end{tabular}

${ }^{a}$ For both readers (B.B., C.M.) and in the $3 \mathrm{D}$ mean thalamus atlas from Krauth et al. ${ }^{10}$

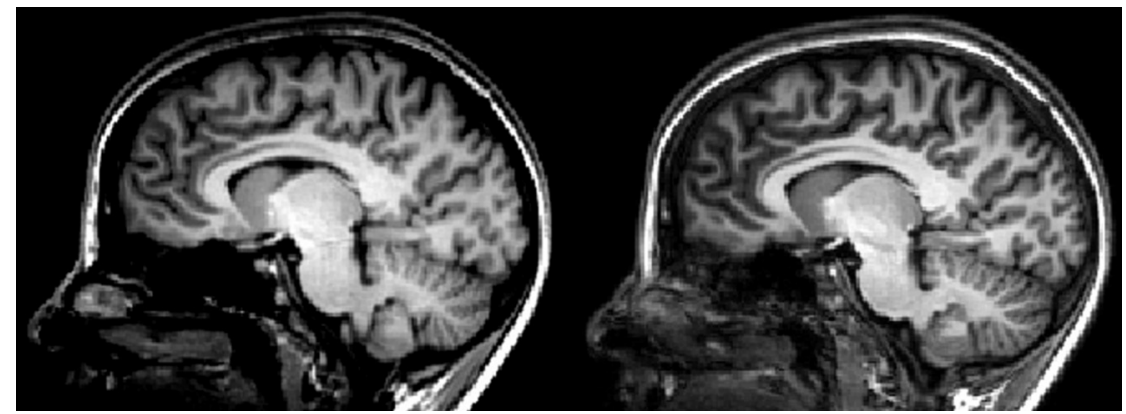

Fig 1. Comparison of the normal MPRAGE protocol (left and middle image, different windowing) with the optimized MPRAGE protocol (right).

ner (Tim Trio; Siemens, Erlangen, Germany) in a 32-channel receiveonly coil. Sequence parameters were as follows: 144 sections; FOV, $256 \times 256 \mathrm{~mm}^{2}$; base resolution, 256 ; section thickness, $1 \mathrm{~mm}$; TE, 3.4 ms; TR, $2300 \mathrm{~ms}$; echo spacing, $8.6 \mathrm{~ms}$; bandwidth, $130 \mathrm{~Hz} /$ pixel; NEX, 2; flip angle, $8^{\circ}$; time of acquisition, 19:39 minutes. The TI was experimentally chosen to maximize suppression of cortical gray matter in the first subject by using low-resolution images. TI was varied in steps of $50 \mathrm{~ms}$ starting at $450 \mathrm{~ms}$ until maximum suppression of cortical gray matter was achieved at TI $700 \mathrm{~ms}$. To visualize the advantages of the optimized sequence in comparison with a standard sequence, an MPRAGE that reflects the local standard for anatomic studies was acquired in 2 subjects with the following parameters: 176 sections; FOV, $256 \times 256 \mathrm{~mm}^{2}$; base resolution, 256; section thickness, $1 \mathrm{~mm}$; TE, $2.96 \mathrm{~ms}$; nonselective inversion pulse with TI, 1100 ms; TR, 2300 ms; echo spacing, 8.5 ms; bandwidth, $130 \mathrm{~Hz} /$ pixel; averages, 2; flip angle, $8^{\circ}$; time of acquisition, 19:39 minutes. In both cases, TE was not freely selectable but was dependent on the other sequence parameters. For labeling of the nuclei, we applied the nomenclature introduced by Hirai and Jones ${ }^{14}$ (Table), which was also used by Morel et al, ${ }^{15}$ because the atlas of Krauth et $\mathrm{al}^{10}$ is based on the Morel atlas.

In the first step, the images were compared with histologic atlases such as the Morel atlas, ${ }^{15}$ the atlas of Mai et al, ${ }^{16}$ and the Schaltenbrand and Wahren atlas. ${ }^{17}$ For further evaluation, the software package 3DSlicer (Version 3.4.1, www.slicer.org) was used. The outlines of the 4 large thalamic nuclei groups (lateral, medial, anterior, and posterior groups) first described by Morel et al (see Table 1 in Krauth et $\mathrm{al}^{10}$ ) were drawn on each section by an experienced neuroradiologist (B.B., 2.5 years of experience). Afterwards, the model-maker module of the software package was used to create 3D models. For the complete thalamus, the sum of the volumes of all 4 thalamic nuclei groups was chosen. For comparison, the mean 3D thalamus atlas published by Krauth et $\mathrm{al}^{10}$ was used. Each image was moved by rigid-body transformation to a standard space with sections parallel to a line between the AC and the PC, with the middle of the AC being the origin. For comparison with the Schaltenbrand and Wahren atlas, the images were transformed into Reil's plane (a tangent line above the AC below the PC). To assess the inter-reader reliability, a second neuroradiologist (C.M., 5.5 years of experience), blinded to the results of the first reader, evaluated all of the datasets, and a consistency score was calculated. Each reader, $n$, selected a volume, $V_{n}$, for each model. The overlap $V_{\mathrm{o}}$ between volume $V_{1}$ and $V_{2}\left(V_{\mathrm{o}}=V_{1} \cap V_{2}\right)$ for each thalamic nuclei group was divided by the larger volume $V_{1}$ or $V_{2}$ $\left[V_{\mathrm{r}}=\max \left(V_{1}, V_{2}\right)\right]$ to form the consistency score $\mathrm{CS}=V_{\mathrm{o}} / V_{\mathrm{r}}$. Both readers were trained in thalamic anatomy. Both readers first studied the myelin stains from the Mai et al atlas ${ }^{16}$ and the Schaltenbrand and Wahren atlas, ${ }^{17}$ as well as the anatomic segmentation of the Morel et al atlas. ${ }^{15}$ Afterward, copies of myelin stains from the Schaltenbrand and Wahren atlas were segmented by hand, and the results were compared with the transparent segmentation overlay provided by the Schaltenbrand and Wahren atlas. For reference, both readers used the anatomic atlases during segmentation.

\section{Results}

The average intercommissural distance was $26.7 \mathrm{~mm}$ (range, 25-29 mm). In the standard MPRAGE protocol, there was a high contrast between white matter, gray matter, and CSF, whereas in the optimized protocol, the contrast between gray matter and white matter was optimal (Fig 1). The outlines of some thalamic nuclei were visible in standard MPRAGE. In contrast, the optimized protocol depicted the borders between the nuclei groups more clearly (Figs 1 and 2). Inverted grayscale images in comparison with myelin stains from histologic sections showed a high level of concordance (Figs 3 and 4). The section position in relation to the midline, the ACPC line, Reil's plane, and the AC (depending on the histologic atlas and 

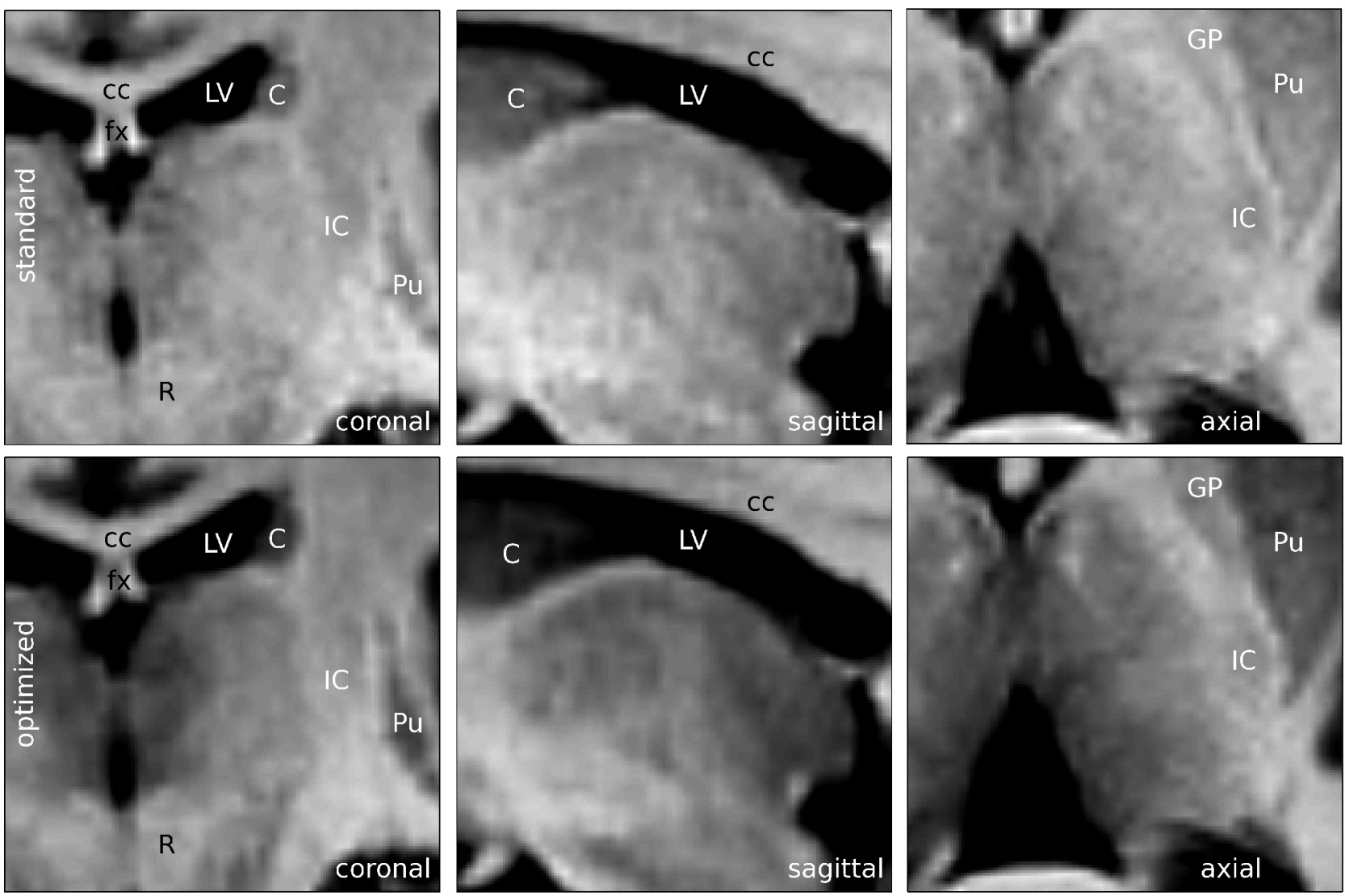

Fig 2. Comparison of the normal MPRAGE protocol (top row) with the optimized protocol (bottom row) in different section orientations. Contrast in both images was chosen for an optimal depiction of thalamus substructures. Adjacent anatomic structures have been labeled. cc indicates corpus callosum; fx, fornix; R, red nucleus; LV, lateral ventricles; C, caudate nucleus; Pu, putamen; IC, internal capsule; GP, globus pallidus.
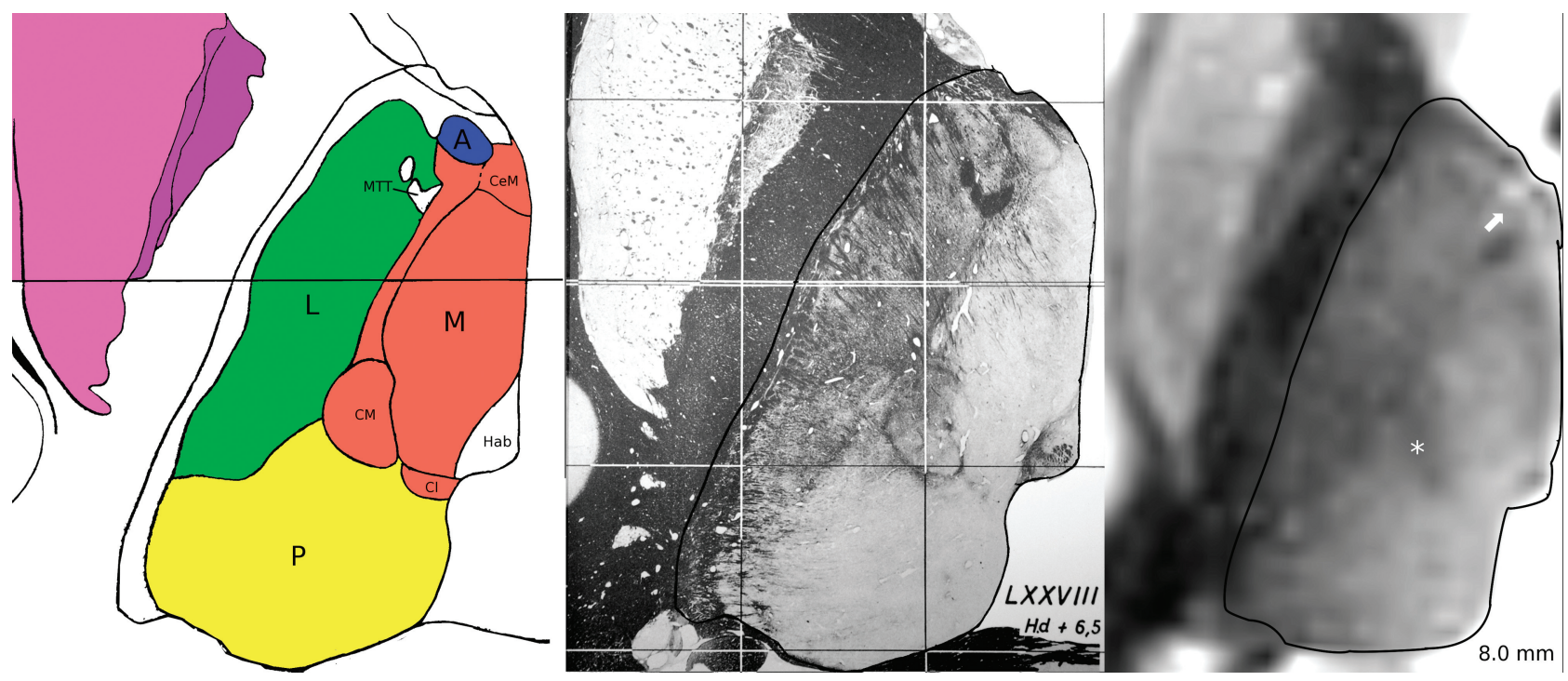

Fig 3. Axial myelin stain (middle) from the Schaltenbrand and Wahren atlas, ${ }_{1}^{17}$ taken it from plate 54 , located 6.5 mm above Reil's plane, and a corresponding inverted gray-scale image from one of the subjects located $8 \mathrm{~mm}$ above Reil's plane (right). For anatomic reference, a schematic drawing adapted from the overlay of the Schaltenbrand and Wahren atlas is shown on the left. The boundaries of the thalamus have been delineated in all images with a thin black line. The white arrow marks the anteroventral nucleus, which can be clearly delineated as a hyperintense structure. The CM (marked by an asterisk) is clearly distinguishable from the surrounding structures. The anatomic structures belonging to the 4 thalamic nuclei groups used in this work have been colored (blue, anterior; red, medial; green, lateral; yellow, posterior).

the orientation) in concordant sections in vivo and in the atlases showed deviations by several millimeters. In Fig 4, the posterior nucleus group is already visible in the in vivo image (dashed line), whereas the histologic section showed no parts of the posterior nucleus group. A vertical line in the axial plane, which represents a parasagittal section, through the MTT in Fig 3, also cuts the medial pulvinar. This suggests that either the plane from the Schaltenbrand and Wahren atlas ${ }^{17}$ in 


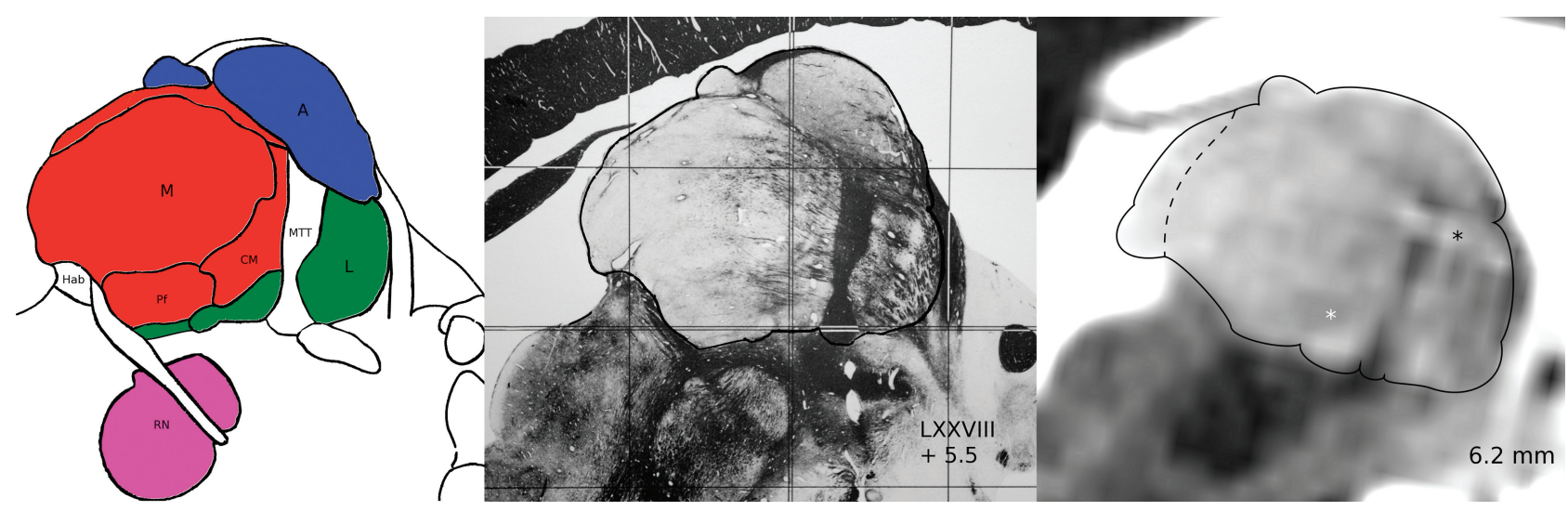

Fig 4. Parasagittal myelin stain (middle) from the Schaltenbrand and Wahren atlas, ${ }^{17}$ taken from plate 44 , located 5.5 mm lateral to the midline and a corresponding inverted gray-scale image from one of the subjects, located $6.2 \mathrm{~mm}$ lateral to the midline (right). The boundaries of the thalamus have been delineated in all images with a thin black line. The white star marks the CM, which can be clearly localized. The bright structure (black star) close to the MTT is a vessel. For anatomic reference, a schematic drawing adapted from the overlay of the Schaltenbrand and Wahren atlas is shown on the left. The anatomic structures belonging to the 4 thalamic nuclei groups used in this work have been colored (blue, anterior; red, medial; green, lateral).

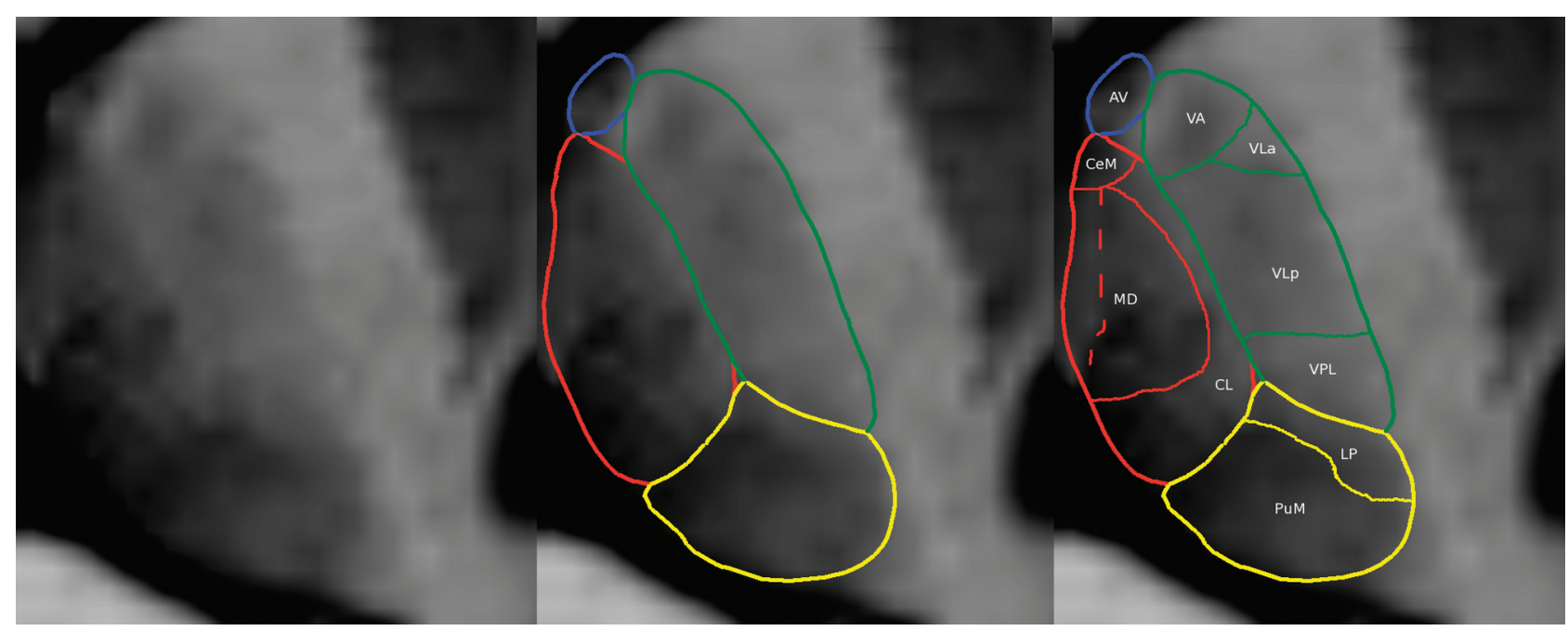

Fig 5. An axial section of the optimized MPRAGE. Note that not only the 4 large nucleus groups (middle image) but also the further substructures can be identified. Medial to the MD, a hyperintense structure is also visible (dashed line depicts border), which is most likely the superficial medial nucleus ${ }^{9}$ not described in the Morel atlas. ${ }^{15}$ In the lateral nucleus group, only thin bands of white matter can be used to distinguish the different parts. The MTT is clearly visible as a hyperintense spot between the anteroventral nucleus (AV), central medial nucleus (CeM), and ventral anterior nucleus (VA). CL indicates central lateral nucleus; VLa, ventral lateral anterior nucleus; VLp, ventral lateral posterior nucleus; VPL, ventral posterior lateral nucleus; LP, lateral posterior nucleus; PuM, medial pulvinar.

Fig 4 is slightly tilted or the MTT in the thalamus used for the sagittal sections of the Schaltenbrand and Wahren atlas had a relatively medial-positioned MTT.

With the myelin stain-like contrast of inverted gray-scale images from the optimized MPRAGE sequence, the borders of the 4 large thalamic nuclei groups could be identified in all subjects and marked by hand. Figure 5 depicts a typical example of the demarcation of the 4 thalamic nuclei groups. The Table summarizes the size of the demarked thalamic nuclei group in comparison with the Krauth atlas. The anterior group was smaller compared with the atlas, while all the other thalamic nuclei groups were larger than those in the atlas. On average, the measured thalami were about $12 \%$ larger than the thalamus of the atlas. The accordance in size, location, and shape of the identified thalamic nuclei groups was high between both raters (Table and Fig 6). For the lateral, medial, and posterior thalamic nuclei groups, the average consistency score between the volumes was $91 \pm 1.3 \%$ / $86 \pm 6.5 \% / 92 \pm$
$1.1 \%$, which was considered very good. For the small anterior thalamic nuclei group, a slightly lower consistency score of $75 \pm 5.9 \%$ between the 2 raters was reached.

\section{Discussion}

This study showed that the main substructures of the thalamus can be identified within a reasonable measurement time of $<20$ minutes in healthy subjects with an optimized acquisition protocol. The shape and localization of the 4 thalamic nuclei groups were in good agreement with the Krauth atlas, whereas the overall size was, in general, larger (Table). An overestimation of the size of the thalamus by the neuroradiologists is unlikely because structures that are easy to identify, such as the MTT, the habenulae, or the pulvinar, could not be matched with the 3D atlas, and the average intercommissural distance $(26.7 \mathrm{~mm})$ was $1.3 \mathrm{~mm}$ larger than the average intercommissural distance $(25.4 \mathrm{~mm}, 23-29 \mathrm{~mm})$ of the 5 brains used for the Krauth atlas. ${ }^{10,15}$ The Morel atlas ${ }^{15}$ was used to 

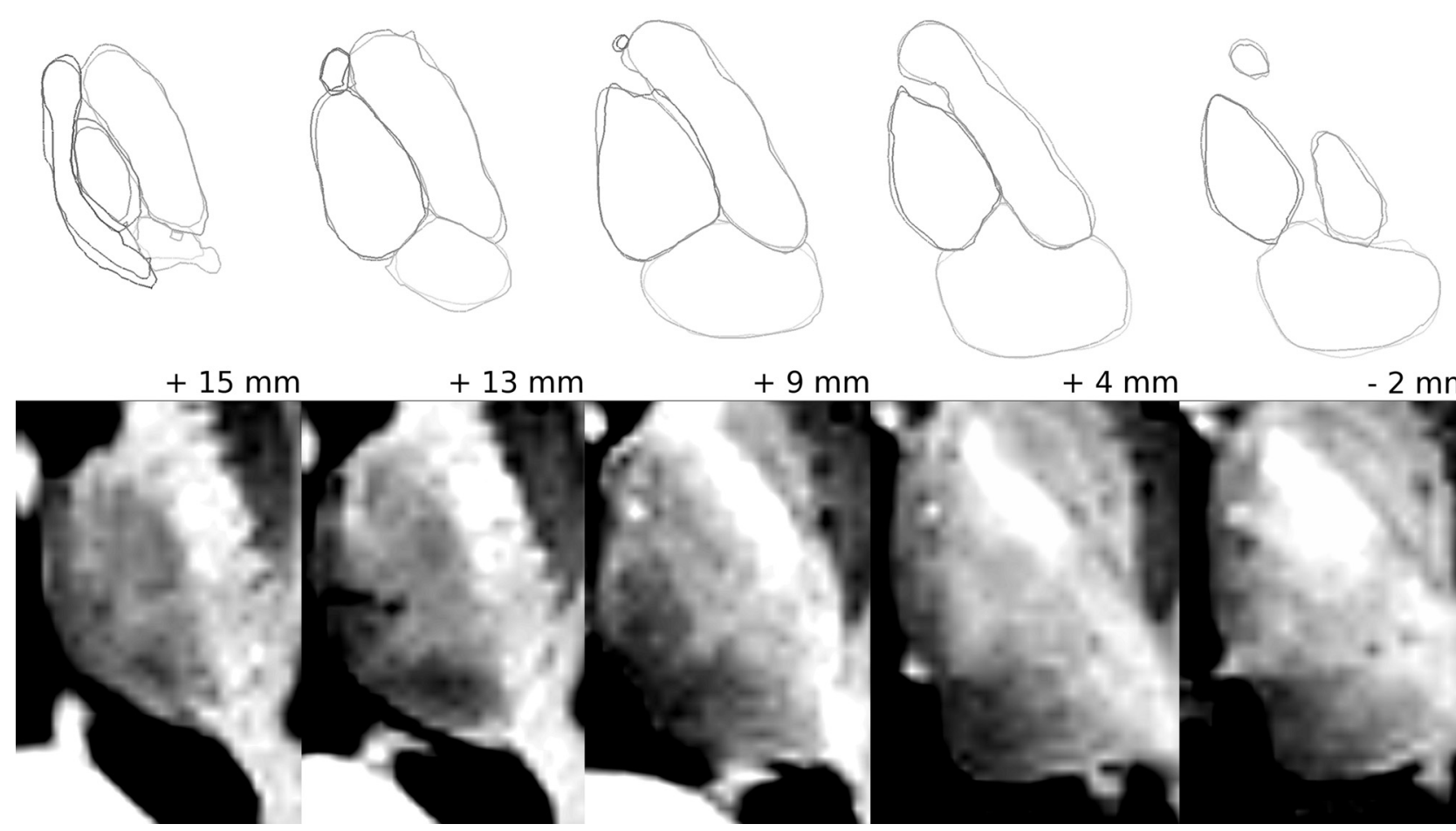

$+4 \mathrm{~mm}$

$-2 \mathrm{~mm}$

Fig 6. Optimized MPRAGE (bottom row) and nucleus groups in axial sections parallel to the ACPC line (from left to right: apical to caudal sections; position given in millimeters relative to the ACPC line) selected by the first and second readers (top row, light and dark gray lines).

create the Krauth atlas, ${ }^{10}$ and it was not corrected for shrinkage due to the fixation process. This explains the average difference of approximately 13\%, and, thus, should be kept in mind by neurosurgeons when planing stereotactic deep brain stimulation implantation. In contrast to the on-average larger size of the thalamus, the identified anterior nucleus group was, in all except 1 subject, smaller than that in the reference atlas. The anterior nucleus group consists of several small nuclei. In particular, the anterodorsal nucleus and the anteromedial nucleus are rather small nuclei, whereas the LD is a thin nucleus. All of the 3 nuclei are, in at least 1 dimension only, approximately 1-3 mm thick and, therefore, consist mainly of voxels that are part of the border of the nuclei. With an isotropic resolution of $1 \mathrm{~mm}^{3}$, partial volume effects at the borders easily explain the difference in size for the anterior group and explain the larger difference in the drawn volumes between the 2 readers in the case of the anterior nucleus group. For the 3 other thalamic nuclei groups, which have a larger volume and, due to their shape, fewer voxels located at the boundaries, the inter-reader agreement was much better.

Although many small substructures of the thalamus were identifiable (Fig 3C), a clear discrimination of all thalamic substructures, possible with longer measurement times of hours or with a higher magnetic field strength, ${ }^{12}$ was not achievable. However, the measurement technique used in this study can be easily applied in presurgical examinations. For a clinical application, the long measurement time of 20 minutes requires a strict prevention of head movement during the scanning time. This can be achieved with sedation or narcosis, which is frequently performed in patients with movement disorders. Although the definite identification of even small substructures was not possible, the suggested protocol clearly de- lineates important substructures and can be used to optimize correlation with stereotactic atlases and navigation planning.

There are several possible solutions to depict even smaller substructures with certainty. Due to the small size of many thalamic nuclei, a higher resolution might be necessary to depict the substructures. If the resolution is increased isotropically, signal intensity to noise would decrease unacceptably or measurement time would increase unacceptably. Because the shape of many nuclei varies slowly in 1 dimension (eg, sagittal or coronal for $\mathrm{LD}$, axial for the $\mathrm{CM}$ ), one might optimize the sequence, depending on the target nucleus. In this case, a low resolution in the slowly varying dimension can be combined with a high resolution in the other 2 dimensions. ${ }^{13}$ Kanowski et $\mathrm{al}^{13}$ suggested using at least an in-plane resolution $0.6 \times 0.6$ $\mathrm{mm}^{2}$ with a 2 - to $3-\mathrm{mm}$ section thickness to depict the CM. Because the various thalamic nuclei differ not only in their transversal but also in their longitudinal relaxation time, a multimodal imaging approach as applied by Deoni et $\mathrm{al}^{11}$ at $1.5 \mathrm{~T}$ might be possible in a reasonable measurement time at higher field strengths. Until now, results from new imaging techniques to segment the thalamus, such as diffusion tensor imaging, ${ }^{18,19}$ could only be compared with histologic atlases. With the new sequence, an individual comparison is possible, which might give a more detailed view inside the thalamus.

Other imaging modalities exist to depict the internal anatomy of the thalamus. Just recently, Abosch et $\mathrm{al}^{20}$ showed high contrast images of the thalamus acquired with SWI at 7T. The measurement time for an isotropic resolution of $0.67 \mathrm{~mm}^{3}$ was 15 minutes. In Fig 6 of the article by Abosch et al, ${ }^{20}$ an axial section of the thalamus at the level of the habenulae is depicted. As in the optimized MPRAGE, many substructures can be identified, though the contrast differs for some substruc- 
tures, while others show a similar contrast in comparison with the optimized MPRAGE protocol. Whether SWI images at 3T provide a similar contrast and if the combination of the different contrasts of T1 and SWI further improves segmentation need further investigation. Gringel et $\mathrm{al}^{21}$ reported that with multiparameter magnetization-transfer maps, improved contrasts can be obtained within the thalamus in a measurement time of 23-25 minutes, but postprocessing is required to calculate the multiparameter maps. The multiecho fast low-angle shot sequence needed is not available as a standard sequence on most scanners; this requirement is currently another drawback for clinical application. Whether the optimized MPRAGE or the multiparameter maps provide a better contrast was not evaluated.

\section{Conclusions}

With the optimized 3D MPRAGE protocol, the main substructures of the thalamus can be depicted. This permits an improved individual targeting for patients undergoing deep brain stimulation electrode implantation, which, before surgery, does not solely rely on stereotactic atlases.

\section{References}

1. Cooper IS, Upton AR, Amin I. Reversibility of chronic neurologic deficits: some effects of electrical stimulation of the thalamus and internal capsule in man. Appl Neurophysiol 1980;43:244-58

2. Lyons KE, Pahwa R. Deep brain stimulation and essential tremor. J Clin Neurophysiol 2004;21:2-5

3. Volkmann J. Deep brain stimulation for the treatment of Parkinson's disease. J Clin Neurophysiol 2004;21:6-17

4. Krauss JK, Yianni J, Loher TJ, et al. Deep brain stimulation for dystonia. J Clin Neurophysiol 2004;21:18-30

5. Fisher R, Salanova V, Witt T, et al. Electrical stimulation of the anterior nucleus of thalamus for treatment of refractory epilepsy. Epilepsia 2010;51:899908

6. Velasco F, Velasco M, Velasco AL, et al. Electrical stimulation of the centrome- dian thalamic nucleus in control of seizures: long-term studies. Epilepsia 1995;36:63-71

7. Weigel R, Krauss JK. Center median-parafascicular complex and pain control: review from a neurosurgical perspective. Stereotact Funct Neurosurg 2004;82: 115-26

8. Schiff ND, Giacino JT, Kalmar K, et al. Behavioural improvements with thalamic stimulation after severe traumatic brain injury. Nature 2007;448: $600-03$

9. Bourgeois G, Magnin M, Morel A, et al. Accuracy of MRI-guided stereotactic thalamic functional neurosurgery. Neuroradiology 1999;41:636-45

10. Krauth A, Blanc R, Poveda A, et al. A mean three-dimensional atlas of the human thalamus: generation from multiple histological data. Neuroimage 2010;49:2053-62

11. Deoni SCL, Josseau JC, Rutt BK, et al. Visualization of thalamic nuclei on high resolution, multi-averaged $\mathrm{T} 1$ and $\mathrm{T} 2$ maps acquired at $1.5 \mathrm{~T}$. Hum Brain Mapp 2005;2:353-59

12. Lemaire JJ, Sakka L, Ouchchane L, et al. Anatomy of the human thalamus based on spontaneous contrast and microscopic voxels in high-field magnetic resonance imaging. Neurosurgery 2010;66:161-78

13. Kanowski M, Voges J, Tempelmann C. Delineation of the nucleus centre median by proton density weighted magnetic resonance imaging at $3 \mathrm{~T}$. Neurosurgery 2010;66(3 suppl operative):E121-23, discussion E123

14. Hirai T, Jones E. A new parcellation of the human thalamus on the basis of histochemical staining. Brain Res Brain Res Rev 1989;14:1-34

15. Morel A, Magnin M, Jeanmonod D. Multiarchitectonic and stereotactic atlas of the human thalamus. J Comp Neurol 1997;387:588-630

16. Mai JK, Paxinos G, Voss T. Atlas of the Human Brain. 3rd ed. Burlington, Vermont: Academic Press; 2007

17. Schaltenbrand G, Wahren H, Atlas for Stereotaxy of the Human Brain. Stuttgart, Germany: Georg Thieme Verlag; 1991

18. Unrath A, Klose U, Grodd W, et al. Directional colour encoding of the human thalamus by diffusion tensor magnetic resonance imaging. Neurosci Lett 2008 434:322-27. Epub 2008 Feb 12

19. Mang SC, Busza A, Reiterer S, et al. Validation of a thalamus segmentation based on local diffusion information. In: Proceedings of the 18th Annual Meeting of International Society for Magnetic Resonance in Medicine, Stockholm, Sweden. May 5-7, 2010:1594

20. Abosch A, Yacoub E, Ugurbil K, et al. An assessment of current brain targets for deep brain stimulation surgery with susceptibility-weighted imaging at 7 Tesla. Neurosurgery 2010;67:1745-56

21. Gringel T, Schulz-Schaeffer W, Elolf E, et al. Optimized high-resolution mapping of magnetization transfer (MT) at 3 Tesla for direct visualization of substructures of the human thalamus in clinically feasible measurement time. $J$ Magn Reson Imaging 2009;29:1285-92 